\title{
Problems and Countermeasures of 3S Technologies Application in Precision Agriculture
}

\author{
Qiulan $\mathrm{Wu}^{1(\bowtie)}$, Zhihong Liu ${ }^{1}$, Dandan Yang ${ }^{1}$, and Zhenrong $\operatorname{Han}^{1}$ \\ ${ }^{1}$ College of Information Science and Engineering, Shandong Agricultural University \\ \{qiulan.wu, zhihong. liu, dandan.yang, zhenrong.han\}@sdau.edu.cn
}

\begin{abstract}
S technologies are the core technologies of precision agriculture. On the basis of deep analysis of the current $3 \mathrm{~S}$ technologies application in precision agriculture, this thesis points out some problems about this application, mainly including incomplete agricultural remote sensing mode, low level of remote sensing interpretation, low standardization and sharing degree of remote sensing, lack of GIS basic data. It also puts forward some suggestions and countermeasures to solve these problems, including improving and expanding agriculture database, researching the mechanism of agricultural remote sensing image interpretation, enhancing standardization of remote sensing data, improving positioning accuracy of farmland operations, attaching importance to integration of new farm machinery and 3S technologies, and applying seamless integration of multi-source spatial data.
\end{abstract}

Keywords: precision agriculture; 3S technologies; application; problems; countermeasures

\section{Introduction}

Precision agriculture is the new trend in agricultural development in the world today. It is a system supported by information technology based on spatial variation and used to implement a set of modern farming operative techniques as well as management in accordance with positioning, timing and quantifying ${ }^{[1]}$. The core technology of precision agriculture is remote sensing, geographic information system and global positioning system (hereinafter 3S technologies for short). In combination with sampling survey, various environment factors (such as soil structure, water content, terrain as well as plant diseases and insect pests) which affect the crop growth are collected and compared with crop yields. Besides, the reasons for different regional yields are analyzed. And different temporal and spatial information about agricultural production is obtained. According to the features of agricultural resources on each plot, positioning control is implemented according to need. And then using modern agricultural machinery to develop intensive and meticulous farming, in order to gain more economic benefit ${ }^{[2]}$. Precision agriculture brings agriculture into the era of data and information and turns to be the main agricultural production form of rationally using agricultural resources, increasing crop yields, reducing production cost and improving ecological environment. To build a conservation-oriented society and realize sustainable development of agriculture, it is necessary to vigorously promote the development of precision agriculture and make full use of the advantages to develop precision agriculture technology system suitable for China ${ }^{[3]}$. With the rapid development of spatial technology and information technology, the 3S technologies are widely used in modern agriculture, promoting the informatization of agricultural production. In recent years, the precision agriculture, with the $3 \mathrm{~S}$ technologies as the core, has drawn much attention. 


\section{Current Situation and Problems of 3S Technologies Application in Precision Agriculture}

\subsection{Current Situation and Problems of RS Technology Application in Precision Agriculture}

\subsubsection{Current Situation and Problems of RS Technology Application in Precision Agriculture}

RS (Remote Sensing) is a kind of polytechnic which involves using sensors at different altitudes to collect electromagnetic wave information about various ground objects on the surface, process the wave information in a reasonable way and summarize the features of the ground objects on the surface, thus completing the recognition and study of various ground objects. In precision agriculture, RS technology is the core technology for obtaining farmland information, thus providing objective, accurate and timely basic farmland information as well as information of dynamic temporal and spatial changes. At present, the application of remote sensing in precision agriculture includes crop identification and classification ${ }^{[4-6]}$, crop nutrition diagnosis and monitoring ${ }^{[7]}$, crop condition monitoring and yield estimation, establishment and application of agricultural remote sensing information model ${ }^{[7-9]}$, agricultural resources monitoring and protection, agricultural disasters monitoring and assessment ${ }^{[8]}$ and so on.

In China, Li Xinchuan used environment and disaster monitoring satellite to construct time series dataset and completed the classification of soybean, corn and rice in Friendship Farm in reclamation area, Heilongjiang Province ${ }^{[4]}$. Besides, on the basis of the multi-temporal TM (Thematic Mapper) images, Cui Wanning calculated the area of winter wheat. Specifically, Cui mainly classified the ground objects by means of decision trees, principal component analysis, tasseled cap and so on as well as calculated the wheat-growing area by means of NDVI (Normalized Difference Vegetation Index) combination threshold value. And the overall classification accuracy is $81.92 \%{ }^{[5]}$. Ding Meihua used multi-temporal environmental satellite images to calculate the sugarcane planting area in Nanning, Guangxi Province, by supervised classification, decision trees classification and multi-temporal NDVI gradual rejecting ${ }^{[6]}$. The application of optical resources remote sensing images in crop type identification is at most, but due to ubiquitous "different spectra characteristics with the same object" and "different objects with the same spectra", the crop classification is often unsatisfactory, and the interpretation accuracy is not high as well.

In terms of agricultural remote sensing information model, on the basis of optimum index-coefficient of correlation method, Cheng Zhiqing selected the best combination of bands of chlorophyll of wheat leaves and used hyperspectral data of best combination of bands to establish forecasting model of chlorophyll content in wheat leaves $^{[7]}$. Li Wenhui established NDWI (Normalized Difference Water Index) information which was suitable for MERSI (Medium Resolution Spectral Imager), in combination with infrared channel NDWI_EX water index expansion model used for flood monitoring ${ }^{[8]}$. Xin Mingyue adopted spectral derivative and statistical analysis techniques and analyzed relationship between hyperspectral reflectance as well as vegetation index and leaf area index to establish the hyperspectral estimation model of rice leaf area index at different growth stages ${ }^{[9]}$. At present, remote sensing information model, based on multispectral remote sensing data as the mainstream, has achieved good results in agriculture. However, some of the description of physiological and ecology process by the models was concise but some was rambling, so the parameter varied much. And many models took a series of assumptions to describe the unknown physiological process, thus reducing accuracy ${ }^{[10]}$. 


\subsubsection{Current Situation and Problems of RS Technology Application in Precision Agriculture}

\subsubsection{Current Situation and Problems of RS Technology Application in Precision Agriculture}

At present, agricultural remote sensing models are numerous, but the accuracy of most models is not high and the applicability is poor. Currently, some of the models stay only at the experimental stage and need large amount of field observation data to be modified. Due to the atmospheric conditions and data quality, the accuracy of some relatively sophisticated models is unable to satisfy the requirements of precision agriculture. In addition, each model has its own application conditions, so the portability and regularity are poor. Different methods of information extraction are usually adopted according to the actual regional situations, for it is difficult to find a common approach ${ }^{[11]}$.

\subsubsection{Low remote sensing interpretation level}

The application of remote sensing in precision agriculture is still at the exploratory stage and many interpretations are not yet mature, such as the study of mixed spectra of kinds of components in the field (crop, soil and so on). Although single interpretation technology of modern remote sensing technology has become mature, mixed spectra study has just started. Therefore, it needs to strengthen the level of interpretation to perfect interpretation system.

\subsubsection{Low standardization and sharing degree of remote sensing data}

Although access to remote sensing images is numerous, except some data free of charge, a large part of high-resolution remote sensing images are expensive. Currently, in China, the expensive and numerous images are not managed under unified rules, which leads to different coordinates, scattered storage and difficult application, thus resulting in extravagant wastage of resources ${ }^{[12]}$.

\subsection{Current Situation and Problems of GIS Application in Precision Agriculture}

\subsubsection{Current Situation and Problems of RS Technology Application in Precision Agriculture}

GIS (Geographic Information System) is a technical system, supported by computer hardware and software, involving collecting, storing, managing, calculating, analyzing, displaying and describing geographical distribution data about the entire or partial surface space of the earth. The technical support from GIS for the precision agriculture is a must and it is a powerful tool which is used to construct the crop accurate management spatial information. Besides, the farmland information is conveyed and processed thorough GIS which is the first step to implement precision agriculture. The application of GIS in precision agriculture mainly includes collection of farmland environment factors information ${ }^{[14,15]}$, analysis and thematic mapping of farmland spatial data, management of farmland spatial data, establishment of management decision supporting system and so on.

Precision agriculture technology is based on farmland environment data collection and analysis ${ }^{[13]}$. Based on GIS, Zheng Lihua developed thematic map models for soil moisture and soil conductivity to guide information sampling of farmland soil ${ }^{[14]}$. Besides, Liu Xiangfeng used 3S technologies to collect information about insect pests on the basis of dynamic GIS technology and provided diagnosis system for real time control $^{[15]}$. In the process of collecting actual farmland information, due to big differences in disaster data and meteorological data, it is hard to obtain precise data.

\subsubsection{Problems of GIS Application in Precision Agriculture}

Precision agriculture relies on large amount of soil fertility data and real-time 
agricultural production factor data, such as data about soil nutrient changes, cropped location disaster data and real-time remote sensing data. Besides, some basic meteorological data is required, such as rainfall, light, and temperature. Due to large differences and restriction from instrument, labor power, financial resources and material resources, it becomes more difficult to obtain the data, thus leading to the lack of GIS basic data ${ }^{[16]}$. What is worse, lack of GIS basic data seriously affects the spatial query and analytical ability of GIS, which hinders the progress of precision work.

\subsection{Current Situation and Problems of GPS Application in Precision Agriculture}

\subsubsection{Current Situation of GPS application in precision agriculture}

GPS (Global Positioning System) is an omnibearing, all-weather, full-time and highprecision navigational satellite system. In precision agriculture, the GPS is widely used in information acquisition and real-time accurate positioning. In addition, realtime positioning accuracy of DGPS (Differential Global Positioning System) is centimeter-sized, so it is widely used in three aspects: agricultural location ${ }^{[18]}$, farm machinery operation navigation and monitoring yields ${ }^{[17]}$. Wei Xinhua used DGPS, GIS and unit operation speed detector to develop site-specific crop management vehicle embedded information processing system. Test result of simulation test showed that the system functioned correctly and could produce distribution map of farmland information and interpret the prescription map. And this system applied for telematics information process of farmland operation machine set of intelligent sitespecific crop management system ${ }^{[17]}$. Li Yongjian developed agricultural automatic navigation system based on the combination of positioning and dual close loop control $^{[18]}$.

\subsubsection{Problems of GPS Application in Precision Agriculture}

Positioning accuracy is a key technology in the development of precision agriculture. The current accuracy of GPS cannot well meet the requirements of precise positioning operation. GPS signal is vulnerable to occlusion, interference and other environmental factors. In addition, high system noise and measured noise exist. Therefore, the accuracy and reliability are lowered. Meanwhile, due to the high expense of GPS application, GPS application is restrained in precision agriculture. Moreover, much new agricultural machinery related with GPS, GIS and RS technologies are imported with high cost, which restricts application and popularization of GPS in agricultural machinery. 


\subsection{Current Situation and Problems of the Application of 3S Integration Technology in Precision Agriculture}

\subsubsection{Current Situation of 3S Integration Technology Application in Precision Agriculture}

In precision agriculture, simply using only one of the GPS, RS and GIS technologies often fails to meet the needs of integrated engineering, nor the need for ground measurement, storage management, information processing and analysis mode in precision agriculture. Therefore, it is necessary to integrate RS, GIS and GPS together, referring to $3 \mathrm{~S}$ integration technologies. In recent years, the domestic study of the integration of RS and GIS is numerous and has become more and more mature. In Dingzhuang Town, Guangrao County, Dongying, Shandong Province, as the objective of study, Li Wenlu Ding constructed and applied agricultural resources information sensing model based on RS, intelligent agricultural resources information management and decision based on GIS, dynamic monitoring of agricultural resources integrated by RS-GIS and perception model of physicochemical properties of soil integrated by GPS-GIS ${ }^{[19]}$. Based on specific use and integrated mode of GIS, RS and GPS, Xu Haitao explored an adapted 3S technological integration frame for the intelligent agricultural machinery integration system. And this frame was combined with internet of things and cloud computing to construct intelligent agricultural machinery integration system with the $3 \mathrm{~S}$ technologies as the core ${ }^{[20]}$.

\subsubsection{Problems of 3S Integration Technology Application in Precision Agriculture}

Although the 3S technologies have been widely applied, most of the content depends on others. So it is hard to completely unify the three technologies, nor realize integrated data management. In terms of $3 \mathrm{~S}$ integration, the most important is the data integration and transformation, that is to say, data consistency, compatibility, and multiple sources. For example, the data conversion in the integration of RS and GIS leads to the requirement of data convertor in updating some software. However, due to the different formats of different systems in different fields and private internal data structure, conversion efficiency of the convertor is low. Ultimately, it is hard to widely apply the $3 S$ integration technology. In addition, because GIS, RS and GPS data are stored separately with different formats, it is hard to comprehensively use the data. 


\section{Suggestions and Countermeasuress}

\subsection{Improve and Expand Agricultural Database}

Agricultural database is the foundation of scientific management for farmland. To maintain the vitality of agriculture database, it is a must to achieve continuous improvement and updating of the database. To carry out thoroughly the research of the standard spectral characteristics in agricultural applications and summarize the spectral variation rules of standard objects in different conditions can improve and expand agriculture database as well as the accuracy and applicability of agricultural remote sensing model ${ }^{[11]}$. In addition, due to the fact that the data stored in $3 \mathrm{~S}$ technologies contain large amount of geo-information, to increase the intensity of mining agriculture information so that these implicit data information can be obtained and make full use of modern information technologies to integrate these data can not only expand agricultural database, but also can solve the problem of lacking GIS basic data.

\subsection{Further Study of Image Interpretation Mechanism of Agricultural Remote Sensing}

The input of the remote sensing interpretation should be increased, and common ground objects interpretation systems, particularly the interpretation system of agricultural remote sensing, should be constructed. Traditional interpretation should be combined with modern information technology as well as the development of GIS and GPS, and the spectrum features of different ground objects in different areas should be taken into interpretation system in order to improve the level of interpretation. Interpretation mark and recognition features of farmland water conditions, fertility conditions, and diseases and pests in remote sensing image should be established to achieve intelligent extraction of crop sign information. The breakthrough of all these key technologies cited above will help interpret the related mechanism of crop growth environment and actual distribution of harvest production, and help quantify the dynamic monitoring of remote sensing and establish quantitative model of crop growth and production forecast, which are of great importance for improving scientific management of agricultural field ${ }^{[21]}$. In addition, the following methods can be adopted to improve interpretation accuracy: extracting various features of remote sensing image and utilizing these features comprehensively for recognition; gradually completing the construction of GIS various subject databases and taking advantage of these GIS data to reduce the uncertainty in automatic interpretation of remote sensing; and establishing expert system suitable for automatic interpretation of remote sensing image to improve the flexibility of automatic interpretation $^{[22]}$.

\subsection{Strengthen the Standardization Construction of Remote Sensing Data}

The satellite data archive center of national earth observation should be planned and constructed as a whole to make the nationally invested spatial infrastructure products fully shared and avoid monopoly between the departments, as well as blockade and inadequate use of data. Integrated applications of remote sensing data from home and abroad should be coordinated. State-backed industries should use china's own satellite data, creating a positive interaction. National public services for non-profits, enterprises and individuals should have the access to satellite remote sensing data at discount or for free. China should introduce stronger supportive measures to encourage and promote the use and application of satellite remote sensing data. In addition, remote sensing database in precision agriculture should be established with satellite remote sensing image data standards and coordinate system of unified standard data. On this basis, vigorously carrying out basic construction of public databases and strengthening the standardization and normalization of remote sensing data will help achieve sharing resources, so as to increase the degree of 
standardization and sharing degree of data.

\subsection{Improve Positioning Accuracy of Farmland Operation}

The following are some methods to improve GPS positioning accuracy: increasing the number of satellites, creating favorable observing conditions, extending observing time and strengthening data processing. Increasing the number of satellites can not only enlarge satellite coverage, but also can increase the number of satellites at observation spot. It is an effective way to increase positioning accuracy and guarantee measurement range of positioning information. Creating favorable observation conditions, including ground monitoring, measuring, and customer terminal equipments, can avoid blinding or disturbing during observation, which will affect the accuracy of observation data. Extending observing time and increasing the number of satellites complement each other. When the number of satellites reaches a certain level, extending observing time can efficiently obtain more observation data and provide more foundation for positioning computation so as to increase GPS positioning accuracy. As to strengthening data processing, positioning accuracy can be increased by a series processing of GPS positioning data. For instance, invalid and disturbing data can be removed by filtering, and valid computed value can be obtained by sampling ${ }^{[23]}$.

\subsection{Attach Importance to the Integration of New Agricultural Machinery and 3S Technologies}

The new agricultural machinery involves the integration of subsystems including RS, GIS and GPS, which is a complex engineering. Through studying the experience in the integration of new agricultural machinery and 3S technologies, the accuracy and liability of various technologies used in sowing, fertilization, weeding, irrigation, ridging and harvest is known. Besides, the merits and demerits of the integration of new agricultural machinery and 35 technologies are assessed and studied. On the basis of the above, new development scheme for new agricultural machinery with advanced technology and economic feasibility is put forward, thus accelerating development speed with less time and expense to develop new agricultural machinery which applies for different farming environment and different crops. This is the key to promote precision agriculture. In addition, the basic agricultural departments should be supported in finance and $3 S$ technologies ${ }^{[24]}$. In combination with the personnel and technical training, 3S technologies and new agricultural machinery could be popularized.

\subsection{Apply Seamless Integration Technology of Multi-source Spatial Data}

Different stored data formatting of GIS and RS bring difficulty to comprehensive utilization of the data. Without data formatting conversion, the multi-source spatial data seamless integration technology ${ }^{[25]}$ as a kind of data integration technology, could get access to advanced space of various data formatting. In addition, this integration technology is featured with direct access, integration of format-independent data and integration of location-independent data. Besides, this kind of integration technology helps to realize $3 \mathrm{~S}$ data integration, thus strengthening multiple analysis across data sources of $3 \mathrm{~S}$ integration technology.

\section{Ackonwledgement}

Funds for this research was provided by the National High-tech R\&D Program (863 Program) (Grant No.2013AA102301) and the innovation subject of Shandong province agricultural application technology. 


\section{References}

1. Chen Zhongjun. Current Research Situation and Development Tendency of Precision Agriculture [J]. Farm Machinery, 2015, 09:114-119.

2. Qin Qiming. The Application of 3S Technologies in Precision Agriculture [J]. Journal China Agricultural Resources and Regional Planning, 2000, 06:58-61.

3. Wang Suzhen, Wu Chongyou. Study on 3S Technologies Applications in Precision Agriculture [J]. Chinese Agricultural Mechanization, 2010, 06:79-82.

4. Li Xinchuan, Xu Xingang, Wang Jihua, Wu Hongfeng, Jin Xiuliang, Li Cunjun, Bao Yansong. Crop Classification Recognition Based on Time-series Images from HJ Satellite [J]. Transactions of the Chinese Society of Agricultural Engineering, 2013, 02:169$176+298$.

5. Cui Fangning, Song Xiaoyu, Sun Baosheng, Wang Jihua. Change Detection of Winter Wheat Area Based on Multi-temporal TM Images [J]. Remote Sensing information, 2012, 05:89-95.

6. Ding Mei hua, Tan Zongkun, Li Hui, Yang Yuhong, Zhang Hangqing, Mo Jianfei, He Li, Mo Weihua, Wang Junhua. Survey Methods of Sugarcane Plant Area Based on HJ-1 Satellite Data [J]. Chinese Journal of Agrometeorology, 2012, 02:265-27

7. Cheng Zhiqing, Zhang Jingsong, Meng Ping, Li YanQuan, Wang Hesong, Li Chunyou. Improvement of Algorithm Used for Extraction Hyperspectral Feature Bands of Vegetation [J]. Transactions of the Chinese Society of Agricultural Engineering, 2015, 12:179-185.

8. Li Wenhui, Yang bin, Huang Yonglin. Study on Remote Sensing Monitoring Technology for Flood Disaster Based on MERSI [J]. Science \&Technology Information, 2012, 32:61$62+65$.

9. Xin Mingyue, Yin Hong, Chen Long, Zhang Meiling, Ren Zhiyong, Miao Jing. Estimation of Rice Leaf Area Index with Different Growth Stages Based on Hyperspectral Remote Sensing Data [J]. Chinese Journal of Agrometeorology, 2015, 06:762-768.

10. Chen Huiming. Discussion on the Application of Remote Sensing Technology in Agriculture [J]. Anhui Agricultural Science Bulletin, 2009, 19:190-192.

11. Yao Yunjun, Qin Qiming, Zhang Zili, Li Baishou. Research Progress of Hyperspectral Technology Application in Agricultural Remote Sensing [J]. Transactions of the Chinese Society of Agricultural Engineering, 2008, 07:301-306.

12. Xie Guoxue, Yang Rujun, Lu Yuan. Advanced Analysis of Agricultural Applications of Remote Sensing Technology [J]. Journal of Guangxi Teachers Education University, 2014, 02:88-96.

13. Shi Guobin. Application of GPS and GIS Technologies in Monitoring System of Precision Agriculture [J]. Hubei Agricultural Sciences, 2011, 10:1948-1950.

14. Zheng Lihua, Li Minzan, Ji Ronghua, Ye Haijian, Wu Caicong, Zhang Junning. Development of Soil Moisture Management Models Based on GIS for Farmland and its Application [J]. Transactions of the Chinese Society of Agricultural Engineering, 2009, S2:13-17.

15. Liu Xiangfeng, Meng Zhijun, Chen Jingping, Wu Wenbo. Design and Implementation of Crop Diseases and Pests Information Collecting and Diagnostic System [J]. Computer Engineering and Design, 2011, 07:2361-2364+2408.

16. Chen Lian, Zhou Weijun, Yu De, Zeng Huayu, Wang Fanrong, Fan Tengfang. Application Progress and Prospect of GIS in Precision Agriculture [J]. Journal of Anhui Agricultural Sciences, 2015, 22:352-354.

17. Wei Xinhua, Dan Zhimin, Sun Hongwei, Zhang Jinmin. Development of Vehicular Embedded Information Processing System for Map-based Precision Farming [J]. Transactions of the Chinese Society of Agricultural Engineering, 2013, 06:142-149.

18. Li Yongjian, Zhao Zuoxi, Gao Junwen, Wu Xiaopeng, Guan Wei. Agricultural Automatic Navigation System Based on DGPS Positioning and Double Closed-loop Steering control [J]. Research of Agricultural Modernization, 2016, 02:387-394.

19. Li Wenlu. Technological Models of Agricultural Resource Information and its Application Mechanism Based on 3S at Township Level [D]. Shandong Agricultural University, 2011.

20. Xu Haitao. Research on Integration System of Intelligent Agricultural Machinery Based on 3S Technologies [D]. Chengdu University of Technology, 2015.

21.Zhang Xu, Wang Gaimei. Application of Remote Sensing Technology in Precision Agriculture [J]. Journal of Green Science and Technology, 2011, 07:211-213. 
22. Qin Qiming. The Problem and Approach in the Auto-interpretation of Remote Sensing Imagery [J]. Science of Surveying and Mapping, 2000, 25 (2): 21-24.

23. Song Qicheng, Tian Daokun. Explore and Enhance the Method of Improving GPS Positioning Accuracy [J]. Managers'Journal, 2015, 18:437.

24. Jiang Huan. Application of GPS Technology in Agricultural Machinery [J]. Agricultural Science \& Technology and Equipment, 2013, 02:75-76.

25. Song Guanfu, Zhong Ershun, Liu Jiyuan, Xiao Lebin. A Study on Seamless Integration of Multi-sources Spatial-data (SIMS) [J]. Progress in Geography, 2000, 02:110-1 
